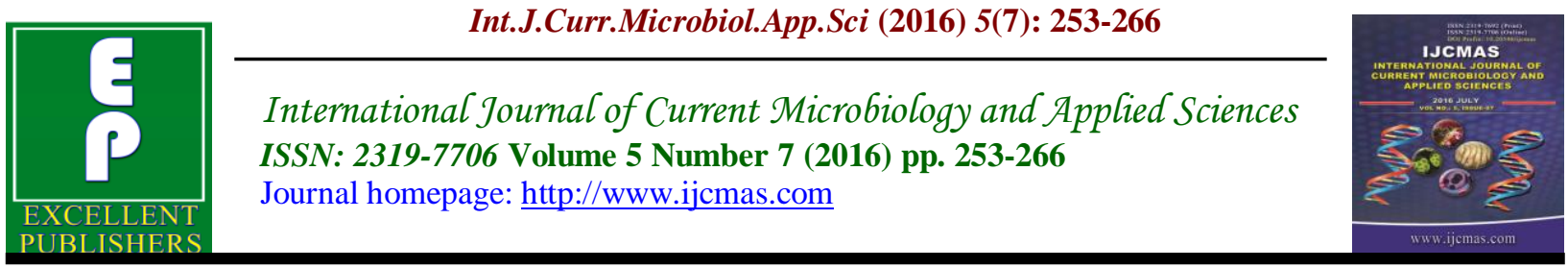

Original Research Article

http://dx.doi.org/10.20546/ijcmas.2016.507.026

\title{
Invitro Assessment of Antimicrobial and Antioxidant Activity of Bioactive Compounds from Marine Algae
}

\author{
S. Rubavathi* and M. Ramya \\ Department of Biotechnology, KSR College of Technology, Tamilnadu, India \\ *Corresponding author
}

\begin{abstract}
A B S T R A C T
Keywords

Biomass,

Bioactive

potential,

Inhibition,

Antioxidant

activity, Drug

Ingredients.

Article Info

Accepted:

12 June 2016

Available Online:

10 July 2016

In recent years, naturally derived metabolites are main target for the treatment of various biological disorders. It is highly used in the medicinal chemistry to avoid side effects in the treatment. In this context, our research focused to derive antibacterial and antioxidant properties from the algal metabolites. Marine algal species such as Chlorella salina, Nannochloropsis oculata, Dunaliella salina and Isochrysis galbanaare focused. The algal species were inoculated in Artificial and F2 natural sea water media. The growth rate was monitored dailyin the media. The maximum growth rate of the algal species was obtained at 10-12 days of incubation time. Mass multiplication was done at small scale reactor vessel $(60 \mathrm{~L})$ to get the maximum yield of biomass. The extracted biomass was dried and used in antibacterial and antioxidant assays. Antibacterial activity done on the four different human pathogens namely Escherichia coli, Klebsiella pneumoniae, Staphylococcus aureus and Proteus vulgaris. The highest inhibitory activity was found in Dunaliella salina. Further, antioxidant potential was done from the potential extract in which Dunaliella salina exhibited the highest activity in DPPH as well as FRAP assays. Inhibitory concentration $\left(\mathrm{IC}_{50}\right)$ of Dunaliella salina was calculated as $80 \mu \mathrm{g} / \mathrm{ml}$ whereas Isochrysis galbana was observed as $100 \mu \mathrm{g} / \mathrm{ml}$. Moreover, chlorophyll, carotenoids and total phenolic content was studied. Results of GC - MS that the crude extracts showed that the crude extract of Dunaliella salina contain Triclosan, Dodecane and $\alpha$-Amyrin which are nowadays used as drug ingredients for the treatment of cancer.
\end{abstract}

\section{Introduction}

Marine algae was one of the largest producers of biomass in the marine environments (Bhadury et al., 2004). Marine micro algae are focused in biomass production due to their ability to survive under environmental stresses like light intensity, dark, heat, UV exposure, nitrogen and also the metabolites production. Some of the marine micro algal species like
Chlorella salina, Isochrysis galbana, Dunaliella salina, and Nanno chloropsis oculata exhibits notable bioactivities like antibacterial,anti-inflammatory, anti-algal, antifungal, analgesics, antioxidant activities which are effective in the prevention of biofouling and have other likely uses, e.g. in therapeutics and nutraceuticals (Smit, 2004). 
Antioxidants are involved in the prevention of cellular damage as these molecules interact with free radicals and end up the reaction. It paves the way for medicinal focus on cancer, cardiovascular, aging, and many diseases because of their tissue protecting effects by neutralization of ROS. Antioxidants compounds can be derived from both natural and synthetic method. There are many synthetic antioxidants like propyl gallate, butylated hydroxytoluene are available commercially but are unsafe and toxic in nature (Madhavi, 1995). Many researchers are interested deriving antioxidant compounds from natural sources (Rankovic et al., 2009).Therefore they are used in food supplements or food ingredients, feed additives, pharmaceutical and cosmetic industry. Algae are also used as fertilizers and medicines.

Chlorella vulgaris possesses high antioxidant capacity and thus could be a potential new source of natural antioxidants (Goiris et al., 2012). It is noted that Isochrysis galbana has rich bioactive compounds than many other algal species (Prakash et al., 2004). Several reports indicate that Dunaliella has anti-oxidative effects (Vanitha et al., 2007). The present study was conducted to evaluate the antibacterial and antioxidant activity of the bioactive compounds from the marine algae.

\section{Materials and methods}

\section{Sample collection and extraction of bioactive compounds}

The marine algae for the present investigational study were obtained from the Central Marine Fisheries Institute (CMFRI), Cochin, Kerala. The algal species were screened based on the growth kinetics. (reference) Mass culturing of the algal species was performed and they were centrifuged at $3000 \mathrm{rpm}$ for 20 minutes. The biomass obtained was extracted using ethanol by ultrasonicator and the extract was air dried to be preserved for further studies.

\section{Carotenoids Extraction}

The carotenoid was obtained by separation of cold acetone extract with petroleum ether solvent in a separating flask. The upper phase containing carotenoids was collected and stored at $20^{\circ} \mathrm{C}$.

\section{Estimation of Chlrophyll and Carotenoid Content}

In order to estimate the chlorophyll and carotenoid content centrifugation was done at $3000 \mathrm{rpm}$ for 15 minutes using $5 \mathrm{ml}$ of micro algal cultures. The pellet was washed with distilled water. After removal of water by centrifugation, it was suspended in acetone and vortexed well. Acetone extract was separated from the cell debris by centrifuging it in 3000rpm for 15 minutes.

Total carotenoid and chlorophyll levels were determined by UV/visible spectroscopy in $80 \%$ acetone by using the Lichtenthaler equation. Beta carotene was measured at $480 \mathrm{~nm}$.

\section{Thin Layer Chromatography analysis of carotenoids}

The qualitative amount of carotenoids in the algal sample was carried out by using Thin Layer Chromatography with mobile phase of methanol and toluene in a ratio $(5: 95) . R_{f}$ values were calculated. By comparing the standard $\mathrm{R}_{\mathrm{f}}$ values for the chosen mobile phase, the carotenoid sample are identified.

$\mathrm{R}_{\mathrm{f}}=$ Distance travelled by the substance/Distance travelled by the solvent. 


\section{Protein Estimation for Algae}

In order to estimate the protein content, $50 \mathrm{ml}$ of sample was centrifuge at 3000rpm for 1-2min. Then the cells are washed with distilled water and spin again at 3000rpm for 2 min. The chlorophyll was extracted by centrifugation and discard it. The pellet was suspended in small amount of water for protein estimation using Lowry et al., 1951. Optical density was measured at $660 \mathrm{~nm}$ using UV-Visible spectrometer (Hitachi U2900).

\section{Effect of Light Intensity on Total Carotene Production}

Light intensity plays important role in controlling the carotene accumulation in algal cells. The effect of light intensity on growth and pigment content of the Dunaliella salina are increased compared to other marine algae. The chlorophyll content per cell decreased and $\beta$-carotene content per cell are increased on the high light intensity.

\section{Total phenolics Determination}

Different concentrations of $50,100,150$, $250,500 \mathrm{mg} / \mathrm{l}$ of gallic acid $(20 \mu \mathrm{l}$ of gallic acid solution at every concentration made, $1.58 \mathrm{ml}$ water, $100 \mu \mathrm{l}$ of folin reagent and $300 \mu 1$ sodium carbonate, $2 \mathrm{ml}$ in total) was used as standard to determine the phenolic concentration of the samples. Then the tubes were mixed and allowed to stand for 1 hour in the dark at room temperature. The absorbance was measured at $765 \mathrm{~nm}$ using spectrophotometer.

\section{Determination of Antibacterial Activity}

The antibacterial activity was tested against four species of bacteria namely Escherichia coli, Proteus vulgaris, Klebsiella pneumoniae and Bacillus subtilis. Kirby
Bauer well diffusion method was administered to test the antibacterial activity and the more effective algal species was selected to test the efficiency against the methicillin-resistant Staphylococcus auerus.

\section{Determination of Antioxidant Activity}

FRAP assay was done according to (Thaipong et al., 2006). 0.2 M phosphate buffer of $\mathrm{pH} 6.6,1 \%$ potassium ferrocyanide, $10 \%$ of trichloroacetic acid and $0.1 \%$ ferric chloride was used to carry out the FRAP assay. L-Ascorbic acid was used as the standard.

DPPH (2,2-diphenyl-2-picrylhydrazyl) assay was performed as per (Vadlapudi et al., 2012). The extracts prepared at different concentrations were added to the $8 \mathrm{ml}$ of $0.004 \%(\mathrm{w} / \mathrm{v})$ stock solution of DPPH in ethanol (95\%). The scavenging activity of--- on the DPPH radical was determined by measuring the absorbance at $517 \mathrm{~nm}$ in spectrophotometer. The DPPH radical scavenging activity was calculated using the following equation:

(Absorbance of control-Absorbance of sample/Absorbance of control)*100

\section{Results and Discussion}

Culturing and screening of algae for bioactive compunds

A total of four isolates such as Chlorella salina, Nannochloro psisocualata, Dunaliella salina and Isochrysis galbana were cultured in laboratory condition at $18^{\circ} \mathrm{C}$ with a light intensity of $0.05 \mathrm{cal} / \mathrm{cm} 2$ min and tested under microscope.

The unialgal cultures were confirmed by microscopic observation at $100 \mathrm{X}$ and the results were presented in figure 4.2. The results showed that the structure of four 
isolates were very unique in nature. Also Chlorella salina and Nannochloropsis oculata were green algae; Dunaliella salina was a halophilic green algae and Isochrysis galbana was observed to be Brown algae.

The growth curve of the four algal species, Chlorella salina, Nannochloropsis oculata, Dunaliella salina and Isochrysis galbana were studied based on the log phase of the samples. Nannochloropsis oculata was ruled out because of their fastest rate of attainment of log phase. The growth pattern was studied at specific optical densities for each algae.

In Isochrysisg albana the optical density was read at 540nm (Drora et al., 1985). The results revealed that I.galbana reached the log phase by 11 to 12 days. The wavelengths for studying the growth of rest of the marine algae were done based on various literatures (Sanjoy et al., 2011, Huang et al., 2011)

\section{Extraction of Marine Algae}

Mass culturing of the algal species were done and they were centrifuged at 3000rpm for 20 minutes. The pellet obtained was extracted using ethanol by ultrasonicator and the extract was air dried and preserved for further studies. The extraction using sonicator was found to be better as it is a time consuming process when compared with Soxhlet extraction as per Ankit et al., 2010 .

\section{Estimation of Chlorophyll and Carotenoid Content}

The total carotene content was high in Dunaliella salina $(2.32 \mu \mathrm{g} / \mathrm{ml})$ compared to other algal species.Next to Dunaliella salina, Isochrysisg albana showed high amount of carotene content but both chlorophyll $\mathrm{a}$ and $\mathrm{b}$ content was found to be high in Isochrysis galbana.

\section{Protein Estimation (Lowry et al)}

Protein content of algal species were much useful to study the stress level of the respective algae with respect to the environmental conditions. In this 2 species Isochrysis galbana and Dunaliella salina were compared and result says that the species 2 has the highest amount of protein expression compared to species 1 which indicates that the stress level of species 2 is higher than the species 1 .

\section{Total Phenolic Determination}

The amount of total phenolics varied. The obtained results do not show a similarity at all the samples. The ethanolic extracts contains more amount of the phenols compared to other algal species. The Isochrysis galbana contains more amount of the phenols in all the solvent extracts and the Nannochlropsis oculata contains low amount of the phenols compared to other species.

Light intensity plays important role in controlling the carotene accumulation in algal cells. The effect of light intensity on growth and pigment content of the Dunaliella salina are increased compared to other marine algae. The chlorophyll content per cell are decreased and $\beta$-carotene content per cell are increased on the high light intensity.

\section{Antibacterial Screening}

The antibacterial activity was studied using two Gram positive and Gram negative bacteria. Chlorella salina showed good zone of inhibition against Escherichia coli and Klebsiella pneumoniae than against Gram positive. Dunaliella salina exhibited activity against both Gram positive as well as Gram negative bacteria. Staphylococcus aureus 
and Proteus vulgaris was inihibited to a larger extend by Isochrysis galbana. A zone of about $12 \mathrm{~mm}$ and $14 \mathrm{~mm}$ was shown by the above two microbes respectively. The positive controls used were Gentamicin, Ampicillin and Trimethoprim and negative control as ethanol used for extraction. It was reported that Chlorella salina and Isochrysis galbana have a considerable antibacterial activity than Dunaliella salina in a study conducted by Srinivasakumar et al., (2009). In contrast to this it was observed that Dunaliella salina exhibited better results than the other two species. The study also revealed that ethanol extract shows good activity against both Gram negative as well as Gram positive bacteria.

\section{Activity of Dunaliella Salina Against Methicillin-Resistant Staphylococcus Aureus}

The three species of marine algae showed good antibacterial activity against two Gram negative as well as two Gram positive bacteria. Of this Dunaliella salina exhibited more inhibition to the growth of Staphylococcus aureus. Thus it was tested against three strains (NP-267, NP-295, NP377) of Methicillin-Resistant Staphylococcus aureus (MRSA). Gentamicin was used as the positive control and the solvent used for extraction was taken as the negative control.

Zone of inhibition was observed between $15 \mathrm{~mm}$ and $19 \mathrm{~mm}$ for the ethanolic extract of Dunaliella salina. Among these three strains NP-267 was inhibited the maximum. It was reported in one of the study carried out by Ohta et al., in 1994 that Dunaliella primolecta, another species of Dunaliella salina showed the presence of antiMethicillin Resistant Staphylococcus aureus substance under optimum culture conditions. Thus the results obtained using the ethanolic extract of Dunaliella salina against MRSA go in line with the finding of Ohta et al.

Analysis of Variance was done for the antibacterial activity of Dunaliella salina against three strains of MRSA. No significant difference was observed during the experiment using the triplicates. Thus it was obtained that Dunaliella salina was a good inhibitor to these three strains of MRSA.

\section{Antioxidant Activity of Dunaliella Salina and Isochrysis galbana}

Antioxidant activity was determined for the two species of marine algae namely Dunaliella salina and Isochrysis galbana based on their activity in the above experiments. The antioxidant property was measured using two assays. The Ferric Reducing Antioxidant Power (FRAP) assay as well as DPPH(2,2-diphenyl-2picrylhydrazyl) assay was carried out with the ethanolic extracts of two species of which Dunaliella salina showed good antioxidant property.

\section{Ferric Reducing Antioxidant Power (Frap) Assay}

Antioxidant property of Dunaliella salina and Isochrysis galbana was done using Ferric Reducing Antioxidant Power (FRAP) assay. This is based on the reducing power of ferric to ferrous form (which has an intense blue color). The reaction was monitored by measuring the absorbance at $700 \mathrm{~nm}$. The reaction is non-specific, in that any half reaction that has redox potential lower that reduce ferric ferrous half reaction, will drive the ferrous (Fe III to Fe II) ion formation. The change in absorbance was directly related to the total reducing power of the electron donating antioxidants present in the reaction mixture. The standard used 
for the FRAP assay was L-Ascorbic acid. With reference to L-Ascorbic acid, Dunaliella salina showed more reducing power than Isochrysis galbana. The standard error bar was also plotted in the graph.

Many literatures revealed the antioxidant nature of Dunaliella salina due to the presence of carotenoid pigment which is absent in Isochrysis galbana. The study carried out by Herrero et al., in 2006 observed that Dunaliella salina is an excellent source of antioxidants. The literatures showed that extraction process using ethanol possessed more efficient for the isolation of antioxidants. Results pointed out that the extracts contained, several different minor carotenoids besides $\beta$ carotene and isomers that seemed to make a contribution to the antioxidant activity of the extracts.

\section{DPPH (2,2-Diphenyl-2-picrylhydrazyl) assay}

The antioxidant activity of Isochrysis galbana and Dunaliella salina were assessed on the basis of the free radical scavenging effect of the stable free radical DPPH as per the method of Kumar et al., 2008. The DPPH method permitted to calculate the electron or hydrogen atomdonating properties of antioxidants and the rate of reaction towards the free radicals. The spectrophotometric technique uses the 2,2-diphenyl-2- picrylhydrazyl free radical (DPPH), which shows a characteristic spectrum with a maximum absorbance close to $517 \mathrm{~nm}$ in methanol. The addition of an antioxidant compound resulted in a decrease of absorbance proportional to the concentration and antioxidant activity of the compound. The discolouration of purple colour of the DPPH was observed after the incubation. Both extracts of the algal species showed an absorbance range within the LAscorbic acid which served as the standard. Of the two test samples the ethanolic extract of Dunaliella salina showed higher antioxidant activity when compared with Isochrysis galbana. DPPH assay is a widely used method to determine the antioxidant property of marine species (Vadlapudi, 2012).

\section{Gas Chromatography - $\quad$ Mass Spectrometry Analysis}

The GC-MS analysis showed various peaks representing the compounds present in the ethanolic extract of the algal species Dunaliella salina. From the graph it was clear that about eight compounds were identified at 5th, 8th, 12th, 16th, 23rd, 28th, 35 th and 40th minutes. Of these one of the highest peak was at 15.97 which corresponds the compound diphenly ether. The derivatives of diphenyl ether shows good antibacterial as well as good anticancer activity. Triclosan, the diphenyl ether derivative is added in the drugs prescribed for patients suffering from a MethicillinResistant Staphylococcus aureus (MRSA) infection as per Roger et al., 2007. Other peaks corresponding to $\alpha$-amyrin, dodecane, tetradecane etc. also shows notable anticancer property. Dodecane is one of the other compounds present in the ethanolic extract of Dunaliella salina is a potent anti proliferative agent which can be used in the treatment of cancer (Filosa et al., 2006). 
Table.1 Activity of Dunaliella salina against methicillin-resistant Staphylococcus aureus

\begin{tabular}{|l|l|l|l|l|}
\hline \multirow{2}{*}{ Bacterial strains } & \multicolumn{4}{|c|}{ Zone of inhibition for ethanolic extract of } \\
\cline { 2 - 5 } & control & $\begin{array}{l}\text { Chlorella } \\
\text { salina }\end{array}$ & $\begin{array}{l}\text { Isochrysis } \\
\text { galbana }\end{array}$ & $\begin{array}{l}\text { Dunaliella } \\
\text { salina }\end{array}$ \\
\hline Escherichia coli & 18 & $10.27 \pm 0.28$ & $10.03 \pm 0.08$ & $11.33 \pm 0.52$ \\
\hline Klebsiella pneumonia & 17 & $12.07 \pm 0.38$ & $11.23 \pm 0.08$ & $12.37 \pm 0.41$ \\
\hline Staphlycoccus aureus & 18 & $7.90 \pm 0.45$ & $8.1 \pm 0.11$ & $8.73 \pm 0.12$ \\
\hline Proteus vulgaris & 15 & 0 & $2.23 \pm 0.14$ & $6.17 \pm 0.12$ \\
\hline
\end{tabular}

Table.2 Antibacterial activity of ethanolic extracts of Dunaliella salina

\begin{tabular}{|l|l|l|l|l|l|}
\hline \multirow{2}{*}{ Bacterial strains } & \multicolumn{2}{|l|}{ Extract(mm) } & Mean \pm SEM & $\begin{array}{l}\text { Positive } \\
\text { control(mm) }\end{array}$ \\
\cline { 2 - 6 } & Trail 1 & Trail 2 & Trail 3 & & \\
\hline NP-267 & 19 & 19.2 & 19.1 & $19.1 \pm 0.8174$ & 20 \\
\hline NP-295 & 18 & 18.1 & 18.2 & $18.1 \pm 0.8174$ & 22 \\
\hline NP-377 & 17 & 17.2 & 17.1 & $17.1 \pm 0.8174$ & 22 \\
\hline
\end{tabular}

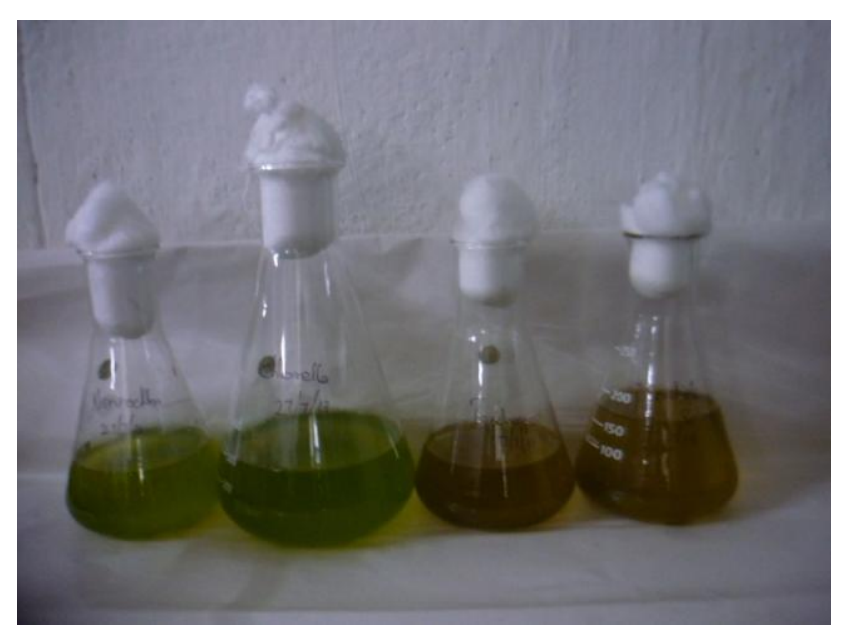

Figure.1 Four species of algal culture

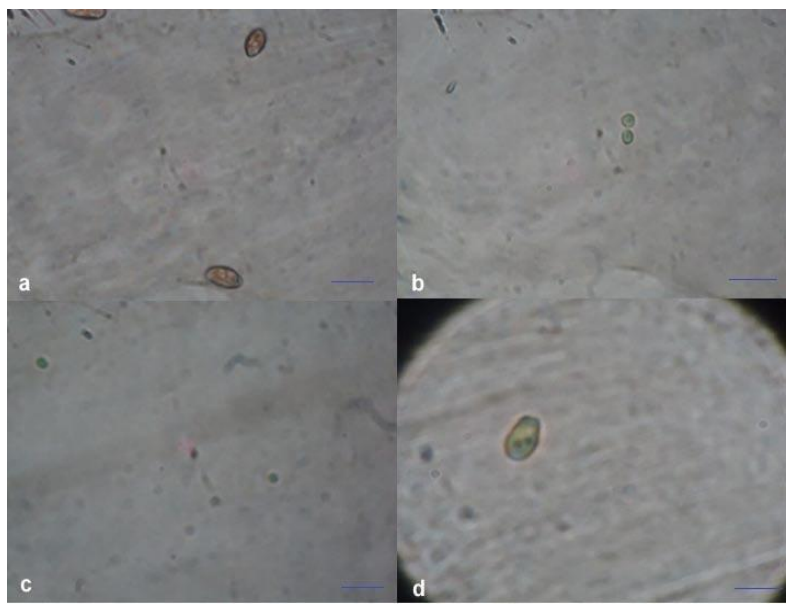

Figure.2 Microscopic images of algae 


\section{Growth Kinetics of various algal species}

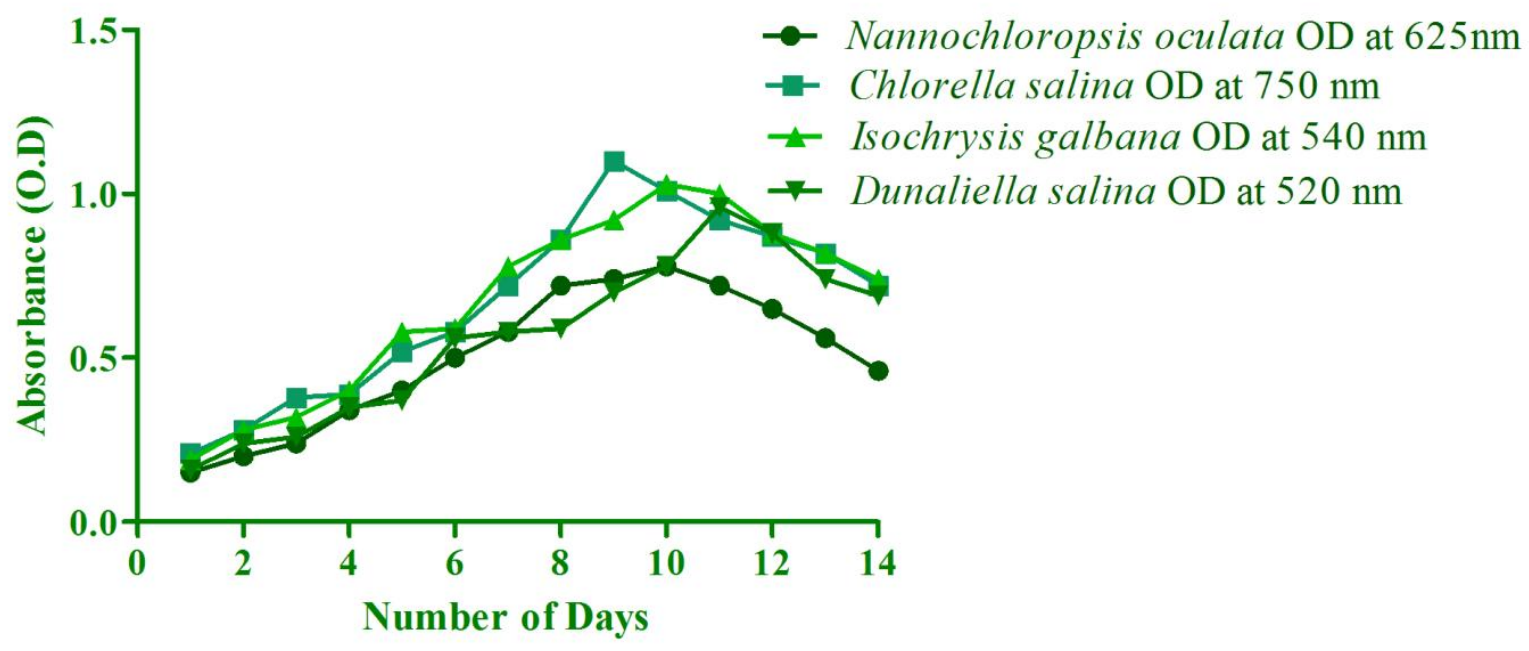

Figure.3 Growth Kinetics Of Various Algal Species.

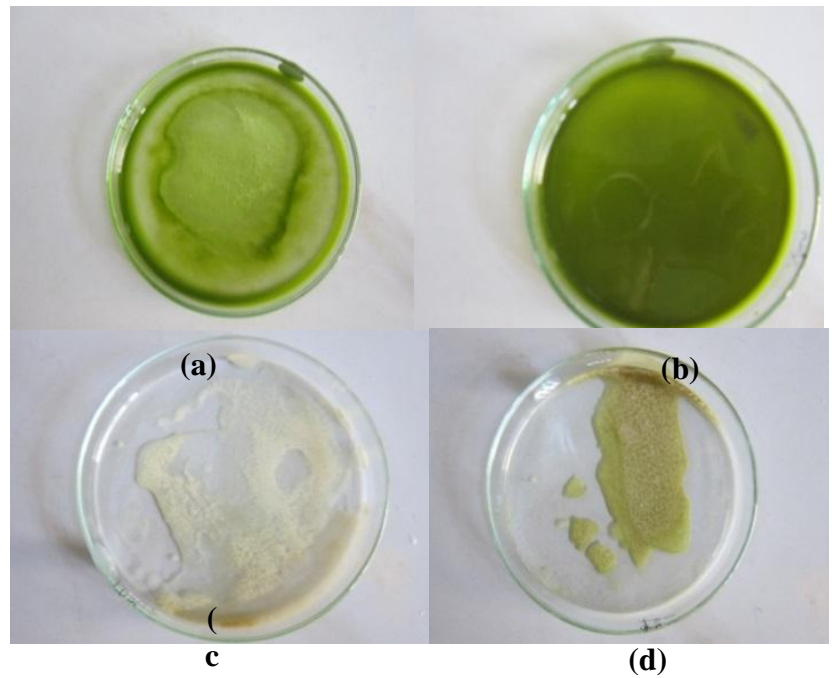

Figure.4 Four species of dried algal extracts

a. Nannochloropsis oculata; b.Chlorella salina; c. Dunaliella salina; d.Isochrysis galbana 


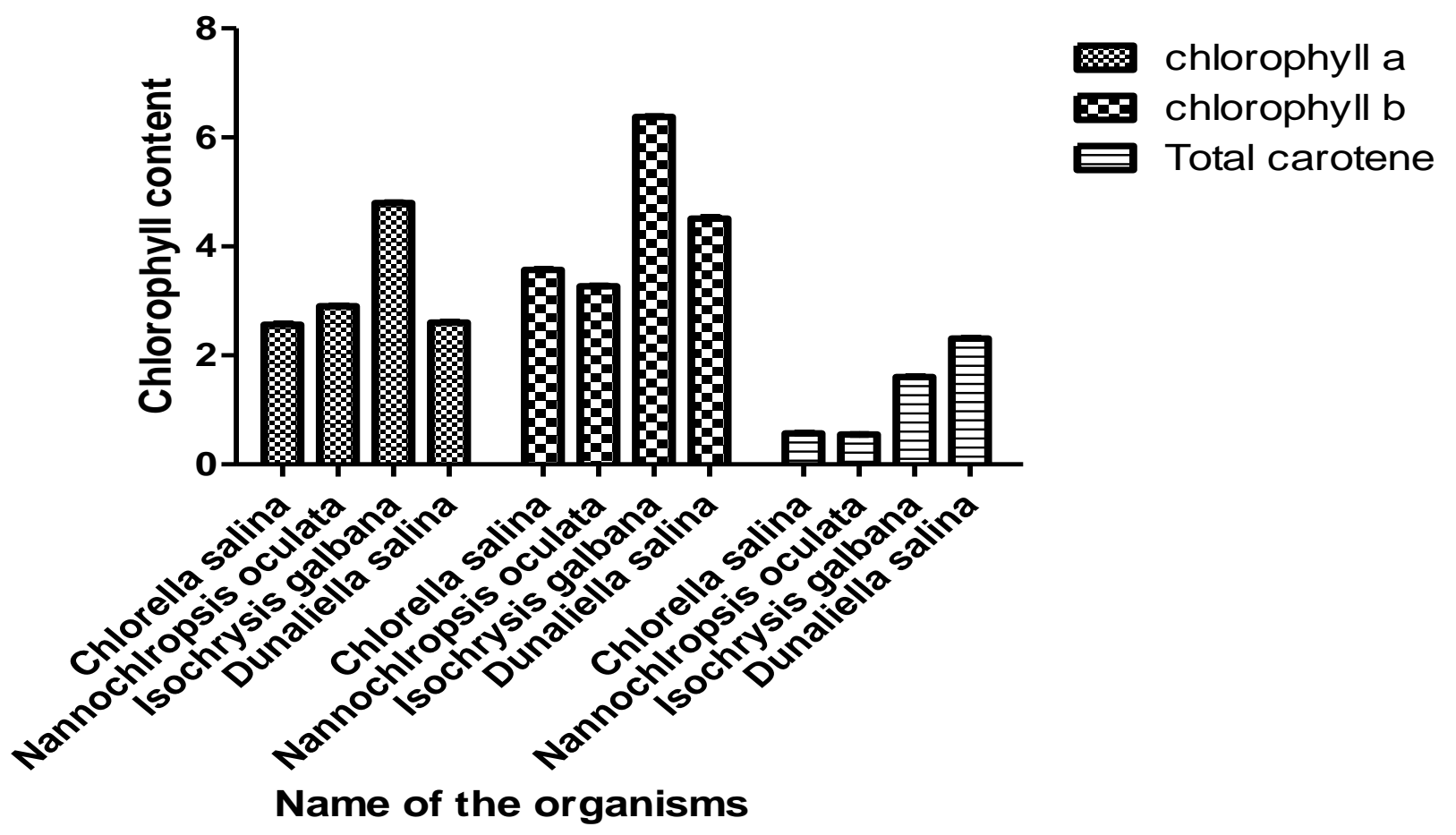

Figure.5 Estimation of chlorophyll and carotenoids in microalgal species

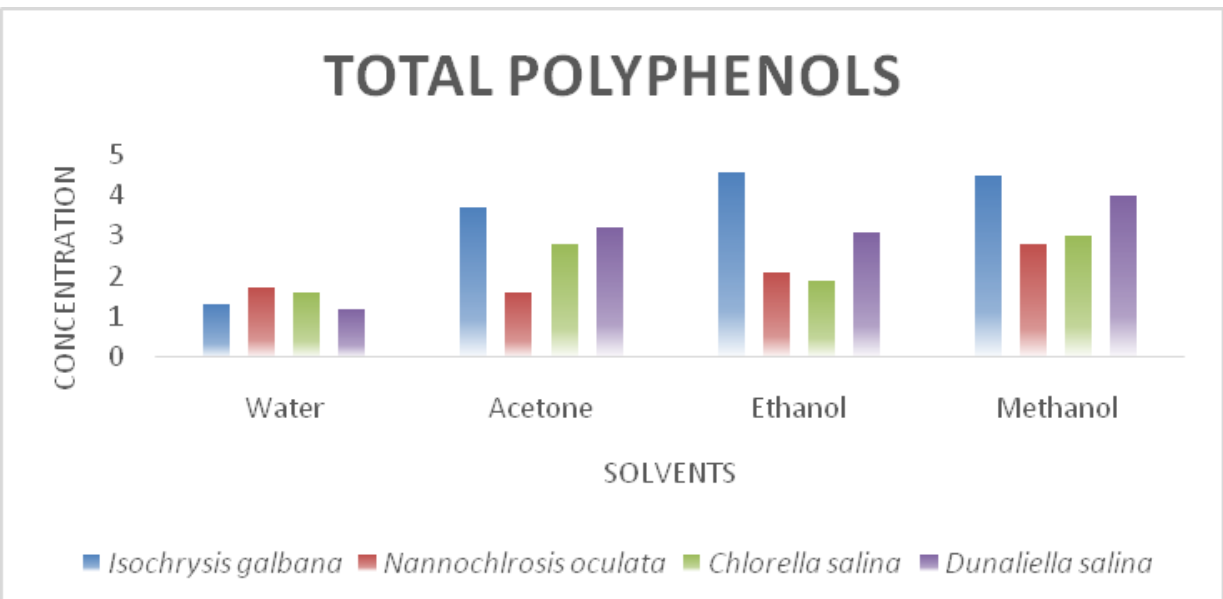

Figure.6 Comparison of Total phenolic compounds in different solvents 


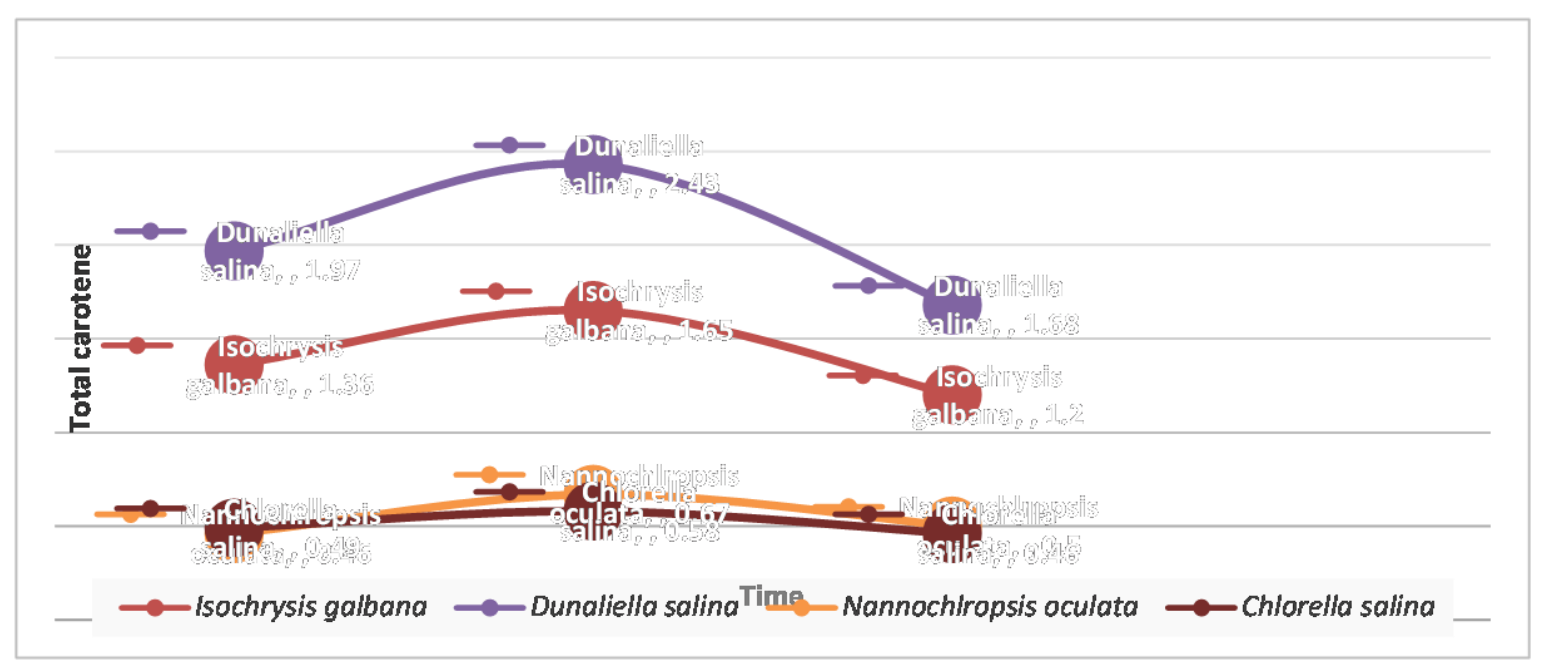

Figure.7 Effect of light intensity on carotene production

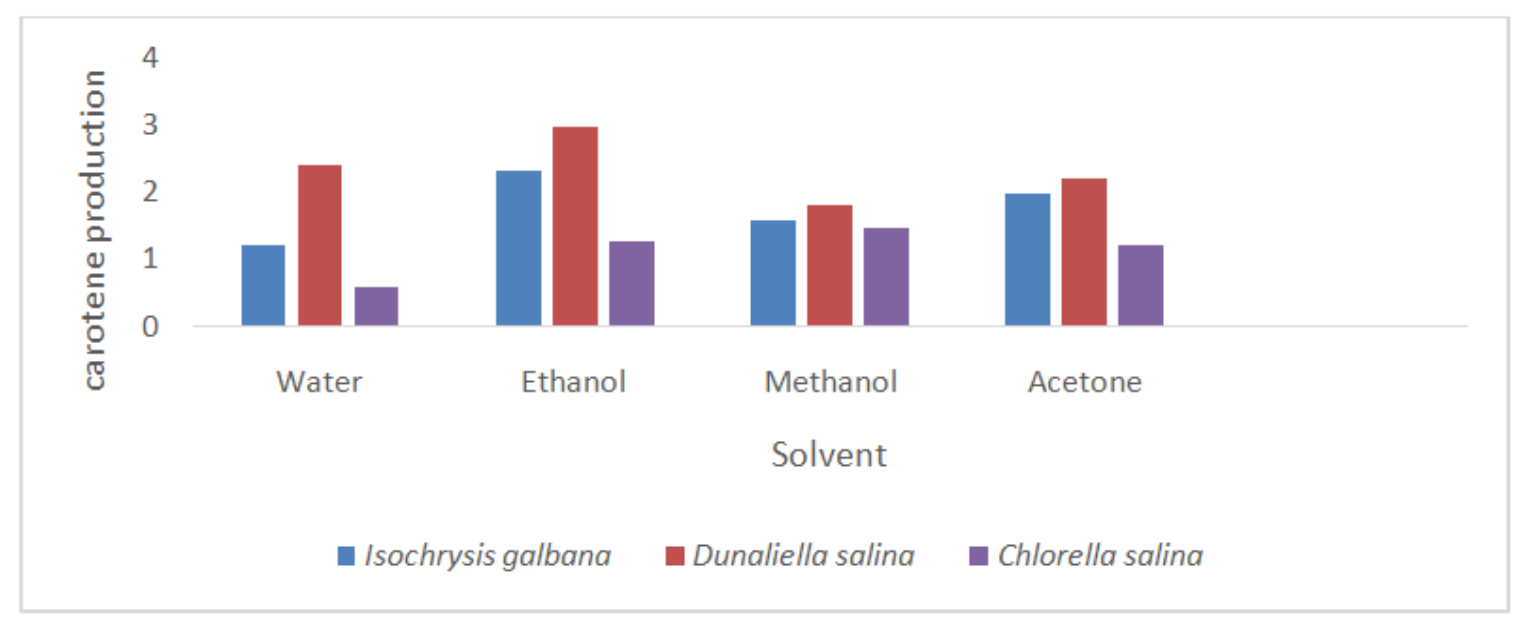

Figure.8 Effect of various solvents on the carotene production

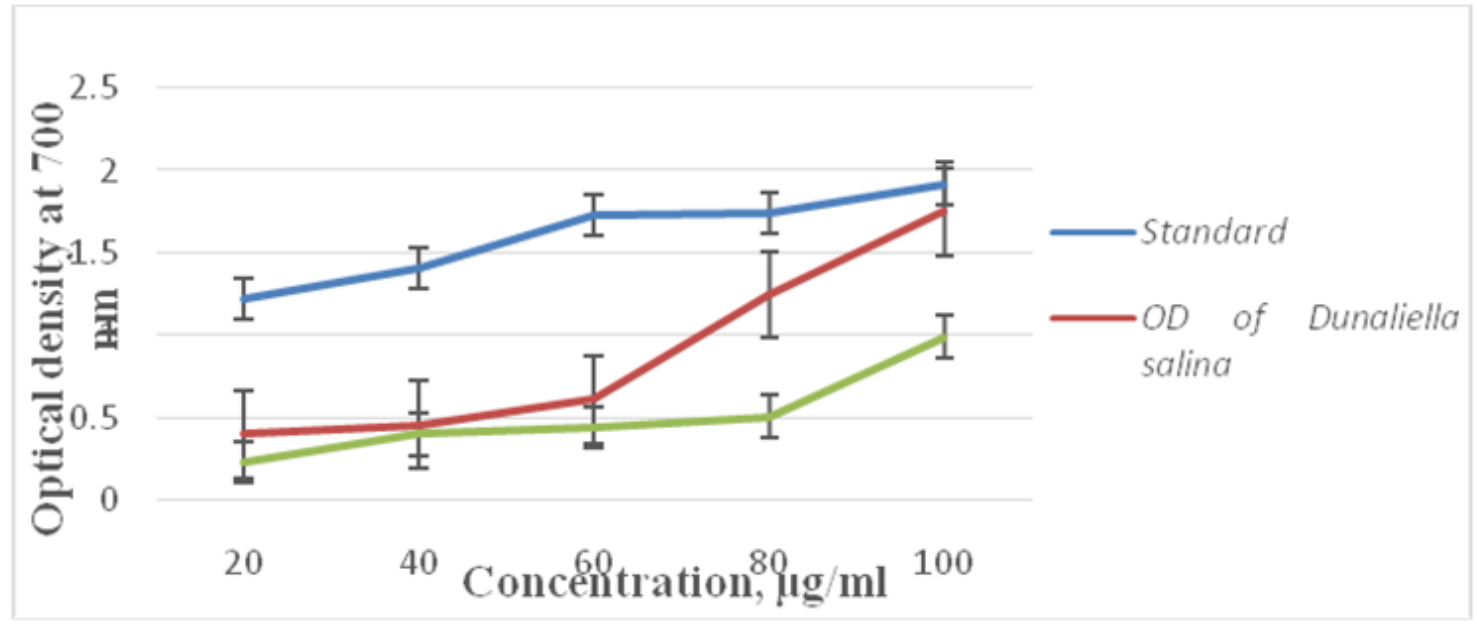


Figure.9 Antioxidant property using FRAP assay

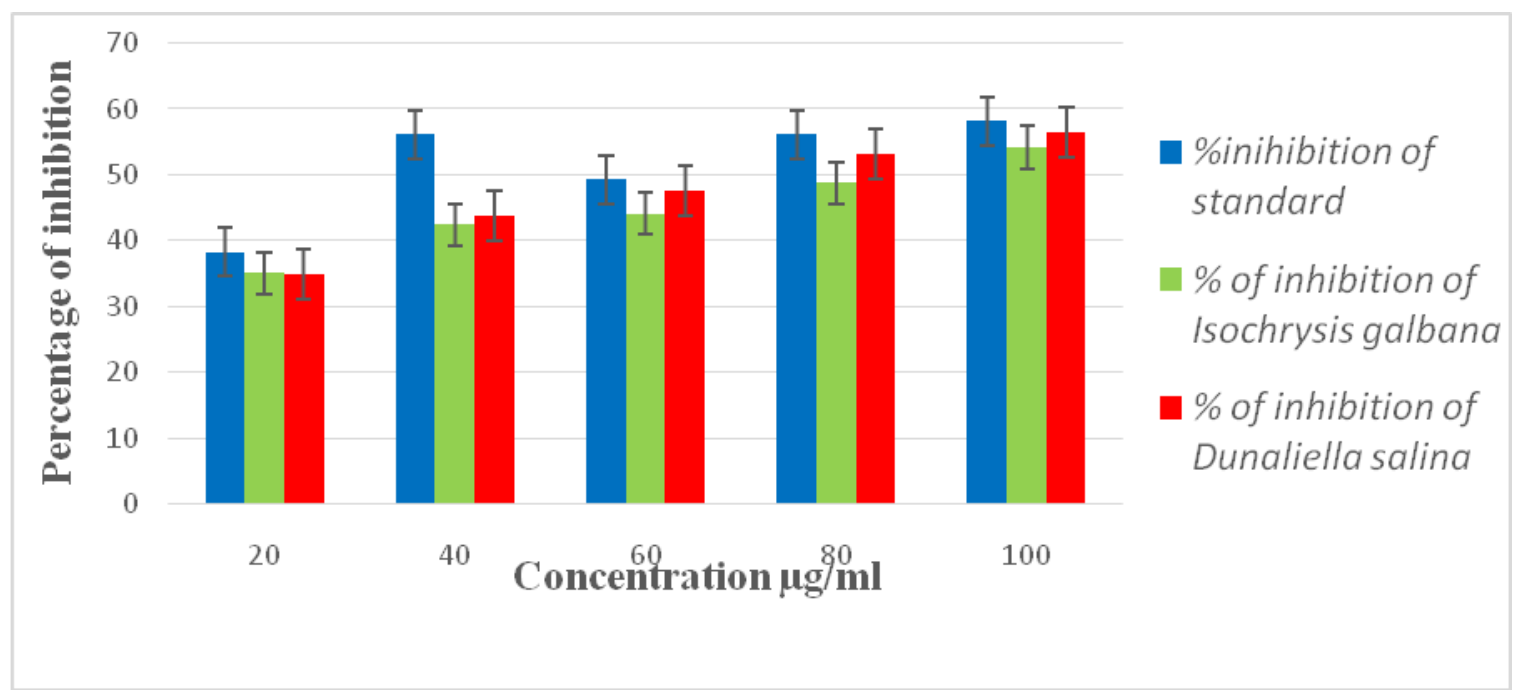

Figure.4 Antioxidant property using DPPH assay

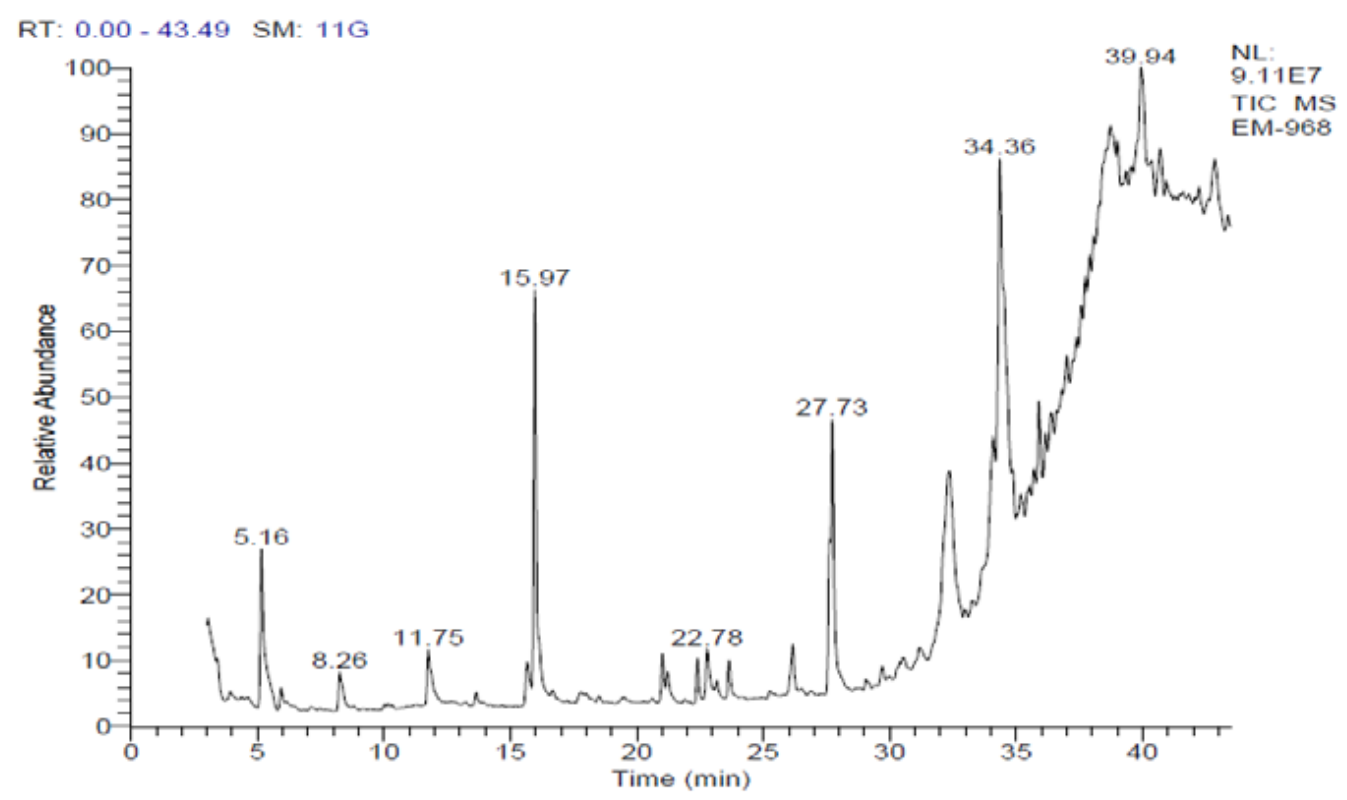

Figure.4.8 Gas Chromatography - Mass Spectrometry analysis

In conclusion, marine microalgae serve as a rich source of the bioactive compounds which can be used in the pharmaceutical field in the place of synthetic drug compounds. Chlorella salina, Isochrysis galbana and Dunaliella salina were extracted with ethanol using Ultrasonication. Based on the results of tests, it can be stated that the microalgal extracts have relatively strong antimicrobial and antioxidant 
properties which requires furthermore studies to determine the antibacterial and antioxidant agent for therapy of various diseases. Dunaliella salina exhibited a remarkable activity against Staphylococcus aureusand it was taken to test against the Methicillin-Resistant

Staphylococcus aureus. Quantitaive analysis for all the above tests were done using the Analysis of Variance (ANOVA).Gas ChromatographyMass Spectrometry (GC-MS) analysis was done for Dunaliella salina which showed better activity in all the tests which showed the presence of volatile compounds. Triclosan is a compound which can be used to inhibit the growth of MRSA culture in the wounds. Further investigation is needed to identify the bioactive compounds in the extracts and isolate and purification of the compounds. Thus Dunaliellasalina is a good source of biologically active compounds and could be used as a substitute for the synthetic drug in future.

\section{References}

Guedes, A.C., H.M. Amaro, F.X. Malcata. 2011. Mar. Drugs, 9: 625-644.

Al-Wathnani, H., Ara, I. and Tahmaz, R.R. 2012 Bioactivity of natural compounds isolated from cyanobacteria and green algae against human pathogenic bacteria and yeast, J. Medi. Plants Res., 6(18): 34253433.

Andricha, G., Nestib, F. And Zinnaia, A. 2005 Supercritical fluid extraction of bioactive lipids from the microalga Nannochloropsis spp., Eur. J. Lipid Sci. Technol., 1(07): 381-386.

Ankit, G., Madhu, N. and Vijay, K. 2010 Modern extraction methods for the preparations of bioactive plant extracts, Int. J. Appl. Natural Sci. (IJANS), 1(1): 8-26.

Bhadury, P. and Wright, P.C. 2004
Exploitation of marine algae: biogenic compounds for potential antifouling applications. Planta, 2(19): 61-578.

Chien, C., Kuod, Y. and Yange, F. 2010 Chemical Composition and Bioactivities of Marine Alga Isochrysisgalbana CCMP 1324 the from Taiwan, Natural Product Communications, 5(1): $1-4$.

Chou, P., Huang, G. and Cheng, H. 2010 Analgesic and Anti-inflammatory activities of an ethanol extract of Dunaliella salina (chlorophyceae), $J$. Food Biochem., 10: 1745-4514.

Doan, T.Y. and Obbard, J.P. 2012 Enhanced intracellular lipid in Nannochloropsis sp. via random mutagenesis and flow cytometric cell sorting, Elsevier Algal Res., 1(1): 17-21.

Drora, K., Zvi, C. And Abeliovich, A. 1985 Optimal Growth Conditions for lsochrysisgalbana", Biomass, 9: 3748.

El-Baz, F.K. and Aboul-Enein, M. 2002 Anticarcinogenic Activity of Algal Extracts , J. Med. Sci., 2 : 243-251.

Filose, R. and Loddo, R. 2006. N, N (4,5dihydro-1h-imidazol-2-yl)3-aza-1,10decane-diamine and N, N(4,5-dihydro1H-imidazol-2-yl)3-aza-1, 10 dodecane-diamine antagonize cell proliferation as selective ligands towards topoisomerase II, J. Pharm. Pharmacol., 58(10): 1415-1420.

Geetha, V. and Venkatachalam, A. 2011 Isolation and Characterization of New Antioxidant and Antibacterial Compounds from Algicolous Marine Fungus CurvulariaTuberculata, Int. Conference on Biosci. Biochem. Bioinformatics, 5.

Gomez, P.I., A. Barriga, A.S. Cifuentes \& M.A. Gonzalez. 2003. Effect of salinity on the quantity and quality of carotenoids accumulated by Dunaliella salina (strain CONC-007) 
and Dunaliella bardawil (strain ATCC-30861) Chlorophyta, J. Biol. Res., 36: 185-192.

Gomez, P.I., M.A. Gonzalez. 2005. The effect of temperature and irradiance on the growth and carotenogenic capacity of seven strains of Dunaliella salina (Chlorophyta) cultivated under laboratory conditions. J. Biol. Res., 38: 151-162.

Goiris, K., Muylaert, K. and Fraeye, I. 2012 Antioxidant potential of microalgae in relation to their phenolic and carotenoid content. J. Appl. Phycol., 1: 10.

Herrero, M. and Jaime, L. 2006 Optimization of the Extraction of Antioxidants from Dunaliella Salina Microalga by Pressurized Liquids, $J$. Agric. Food Chem., 54: 5597-5603.

Horincar, V., Parfene, G. and Bahrim, G. 2011 Evaluation of bioactive compounds in extracts obtained from three romanian marine algae species", AC Romanian Biotechnol. Lett., 16(6).

Hsu, H. and Jeyashoke, N. 2009 Immunostimulatory Bioactivity of Algal Polysaccharides from Chlorella pyrenoidosa Activates Macrophages via 3 Toll-Like Receptor 4", J. Agric. Food Chem., 10.

Huang, W. and Dong, B. 2011 Growth effects on mixed culture of Dunaliella salina and Pheaodactylum tricornutum under different inoculation densitiesand nitrogen concentrations", African J. Biotechnol.,10(61): 1316413174.

Kim, K. and Cha, S. 2012 Neuroprotective Effects of Nannochloropsis oculata Against AAPH-induced Oxidative DNA Damage in HT22 Cells, Int. J. Pharmacol., 8: 527-534.

Kumar, S., Kumar, D. and Saroha, K. 2008 Antioxidant and free radical scavenging potential of Citrullus colocynthis (L.) Schrad methanolic fruit extract, Acta. Pharm., 58: 215220.

Lazarus, S. and Bhimba, V. 2008 Antibacterial activity of marine microalgae against multidrug resistant human pathogens, Int. J. Applied Bioengi., 2(1): 16-22.

Luis, J., Gómez, V. and Irma, E. 2010 Antibacterial and anticancer activity of seaweeds and bacteria associated with their surface, Revista de Biología Marina Oceanografía, 45(2): 267-275.

Pise, N., Jena, K. and Maharana, D. 2010 Free radical scavenging potential, reducing power, phenolic and biochemical constituents of Porphyra species from India, J. Algal Biomass Utln., 1(3): $29-42$.

Rainer, B.V. 2005. Screening of microalgal culture media for the presence of algicidal compounds and isolation and identification of two bioactive metabolites, excreted by the cyanobacteria Nostocinsulare and Nodularia harveyana, J. Appl. Phycol., 17: 339-347.

Roger, B. and Toni, S. 2007 Triclosan resistance in methicillin-resistant Staphylococcus aureus expressed as small colony variants: a novel mode of evasion of susceptibility to antiseptics. J. Antibacterial Chemother., 59(5): 848-853.

Saidani, K., Bedjou, F. and Benabdesselam, F. 2012 Antifungal activity of methanolic extracts of four Algerian marine algae species, African $J$. Biotechnol., 11(39): 9496-9500.

Sakoulas, G. and Robert, C. 2006 Adaptation of Methicillin-Resistant Staphylococcus aureus in the Face of Vancomycin Therapy, Journal of the Royal Society of Tropical Medicine and Hygiene. 42(1): 40-50.

Sanjoy, B. and Hew, W. 2011 Growth and 
proximate composition of tropical marine Cheatoceroscal citrans and Nannochloropsis oculata cultured outdoors and under laboratory conditions, African J. Biotechnol., 10(8): 1375-1383.

Shanab, S.M. and Mostafa, S.S. 2012 Aqueous extracts of microalgae exhibit antioxidant and anticancer activities, Asian Pacific J. Trop. Biomed., 2: 608-615.

Smit, A.J. 2004. Medicinal and pharmaceutical uses of seaweed natural products, J. Appl. Phycol., 16: 245-262.

Srinivasakumar, K.P. and Rajashekhar, M. 2009 In vitro studies on bactericidal activity and sensitivity pattern of isolated marine microalgae against selective human bacterial pathogens", Indian J. Sci. Technol., 2(8).

Taskin, E. and Caki, Z. 2010. Assessment of in vitro anti-tumoral and antimicrobial activities of marine algae harvested from the eastern Mediterranean sea", African J. Biotechnol., 9(27): 42724277.

Thaiponga, K. and Boonprakob, U. 2006. Comparison of ABTS, FRAP, DPPH and ORAC assays for estimating the antioxidant activity from guava fruit extracts, J. Food Composition and Analysis, 19: 669-675.

Vanitha, A., Murthy, K.N. and Kumar, V. 2007. Effect of the carotenoidproducing alga, Dunaliella bardawil, on $\mathrm{CCl}_{4}$-induced toxicity in rats, Int. $J$. Toxicol., 26(2): 159-167.

Yua, C.C., Chenb, H.W. and Chenb, M.J. 2010. Chemical Composition and Bioactivities of the Marine Algae Isochrysis galbana, Natural Product Communication, 5: 1-4.

\section{How to cite this article:}

Rubavathi, S., and Ramya, M. 2016. Invitro Assessment of Antimicrobial and Antioxidant Activity of Bioactive Compounds from Marine Algae. Int.J.Curr.Microbiol.App.Sci. 5(7): 253266. doi: http://dx.doi.org/10.20546/ijcmas.2016.507.026 